\title{
Interacción Endometrio Trofoblasto, en la Implantación Humana: Revisión de la Literatura
}

\author{
Endometrium-Trophoblast Interaction in Human Implantation: Review of Literature
}

\begin{abstract}
Ruth Prieto Gómez ${ }^{1,7}$; Nicolás Enrique Vidal Seguel ${ }^{2,7,10}$; Rodrigo Alberto Lizama Pérez ${ }^{3,7}$; Ricardo Esteban Miranda Krause ${ }^{4,5,7}$; Néstor Eusebio Nahuelcura Millán ${ }^{6,7}$; Marcelo Eduardo Ortega Silva ${ }^{7}$;icolás Ernesto Ottone ${ }^{8,9}$ \& Mariana Deppe ${ }^{6,7}$
\end{abstract}

PRIETO, G. R.; VIDAL, S. N. E.; LIZAMA, P. R. A.; MIRANDA, K. R. E.; NAHUELCURA, M. N. E.; ORTEGA, S. M. E.; OTTONE, N. E. \& DEPPE, A. M. Interacción endometrio trofoblasto, en la implantación humana: Revisión de la literatura. Int. J. Morphol., 37(2):397-405, 2019.

RESUMEN: Un embarazo exitoso requiere de una serie de interacciones mediadas por factores hormonales, moleculares y fenómenos de inmunomodulación. Una de estas interacciones es la que ocurre entre el endometrio y el blastocito, previo y durante el proceso de implantación. El objetivo de esta revisión bibliográfica es complementar lo descrito en la literatura clásica de embriología humana sobre interacción de endometrio-blastocito. La búsqueda bibliográfica se realizó en la base de datos MEDLINE usando los términos en inglés "implantation", "endometrium" y "embryo"; además se realizó una búsqueda manual, que incluyó artículos de revistas no indexadas, libros de texto y atlas. Se consideraron criterios de inclusión y exclusión para la selección de los artículos y otros recursos bibliográficos. Entre los criterios de inclusión se consideraron estudios realizados en humanos, artículos de revisión y experimentación, publicados en los últimos 5 años. Como criterios de exclusión se consideraron artículos que utilizaran animales, estudios sobre fertilidad in vitro, patologías asociadas y artículos no relacionados al tema. Una vez completada la selección, se examinaron los textos completos, en los cuales se aplicaron nuevamente los criterios de exclusión. La búsqueda arrojó un total de 560 artículos, cuyo análisis de los títulos y resúmenes resultó en 475 trabajos excluidos, a partir de los diferentes criterios de exclusión antes descritos. Por lo tanto, se obtuvieron 85 artículos, en los cuales se realizó el análisis del texto completo. De estos artículos, se obtuvieron un total de 34 estudios y los contenidos seleccionados en esta revisión fueron: Endometrio, Interacción endometrio trofoblasto, Aposición, Adhesión y Migración-Invasión. Durante la implantación se genera una interacción entre el endometrio y el trofoblasto, con la participación de moléculas reguladoras de proliferación y diferenciación, como factores hormonales, moleculares y de expresión génica. Sin embargo, los mecanismos específicos de acción e interacción deben continuar siendo investigados, para responder interrogantes en el ámbito del crecimiento y desarrollo humano.

PALABRAS CLAVE: Implantación; Endometrio; Trofoblasto.

\section{INTRODUCCIÓN}

Un embarazo exitoso requiere de una serie de interacciones mediadas por factores hormonales, moleculares y fenómenos de inmunomodulación. Una de estas interacciones es la que ocurre entre el endometrio y el blastocito, previo y durante el proceso de implantación (Moore et al., 2016).
El endometrio experimenta variaciones en su disposición desde la pubertad hasta la menopausia. Los cambios que transcurren durante el ciclo reproductivo o periodo fértil de la mujer, se desencadenan de forma cíclica y variable, con una duración promedio de 28 días (Sadler, 2012). Todo esto, bajo el control hormonal del ovario. Durante este ciclo

\footnotetext{
${ }^{1}$ Departamento de Pediatría y Cirugía Infantil, Facultad de Medicina, Universidad de La Frontera, Temuco, Chile.

2 Escuela de Enfermería, Facultad de Salud, Universidad Santo Tomás, sede Los Ángeles, Chile.

${ }_{3}^{3}$ Departamento de Ciencias Morfológicas, Facultad de Medicina y Ciencia, Universidad San Sebastián, General Lagos 1140, Valdivia 5090000, Chile.

${ }^{4}$ Departamento de Ciencias Morfológicas, Facultad de Medicina y Ciencia, Universidad San Sebastián, Lago Panguipulli 1390, Puerto Montt 5501842, Chile.

${ }^{5}$ Universidad de Los Lagos, Facultad de Salud, Carrera de Kinesiología,Camino a Chinquihue s/n Km 6, Puerto Montt, Chile.

${ }^{6}$ Departamento de Ciencias Básicas, Facultad de Medicina, Universidad de La Frontera, Temuco, Chile.

7 Programa de Magíster en Ciencias, Mención Morfología, Facultad de Medicina, Universidad de La Frontera, Temuco, Chile.

${ }^{8}$ Laboratorio de Plastinación y Técnicas Anatómicas, Centro de Investigación en Ciencias Odontológicas (CICO), Departamento de Odontología Integral

Adultos, Facultad de Odontología, Universidad de La Frontera, Temuco, Chile.

${ }_{9}$ Centro de Excelencia en Estudios Morfológicos y Quirúrgicos (CEMYQ), Facultad de Medicina, Universidad de La Frontera, Temuco, Chile.

${ }^{10}$ Becario CONICYT-PCHA/MAGISTER NACIONAL/ 2017-2217007
} 
menstrual, el endometrio pasa por tres fases funcionales: fase proliferativa; estrogénica o folicular; fase secretora; progestacional o lútea; y la fase menstrual (Eynard et al., 2011; Arteaga et al., 2013). La fase proliferativa se desarrolla bajo la influencia de los estrógenos y paralelamente al crecimiento de los folículos ováricos. La fase secretora se inicia alrededor de dos a tres días después de la ovulación, en respuesta a la progesterona producida por el cuerpo lúteo. En esta fase las glándulas y arterias uterinas se vuelven tortuosas, el tejido congestivo y el endometrio se caracteriza por estar compuesto por tres capas: capa compacta, superficial; capa esponjosa intermedia (ambas constituyen la capa funcional); y una capa basal delgada o submiometrial, encargada de regenerar el endometrio para futuros ciclos (Gratacos et al., 2008; Gómez, 2013). Si no se produce la fecundación, se inicia el desprendimiento del endometrio (capas compacta y esponjosa), lo cual señala el inicio de la fase menstrual. Por el contrario, en caso de fecundación, el endometrio participa en la implantación del blastocito y contribuye a la formación de la placenta (Carlson, 2009; Eynard et al., 2011; Arteaga et al.; Moore et al., 2016).

Previo a la implantación el embrión en estado de mórula rodeado por la zona pelúcida ingresa desde la tuba uterina a la cavidad del útero. Luego, aproximadamente 4 días después de la fecundación, por ingreso de líquido a la mórula, se forma un espacio denominado cavidad blastocística o blastocele, denominándose al embrión en esta etapa blastocisto (Carlson; Arteaga et al.). A medida que ingresa líquido a la cavidad blastocística, las células se separan en dos tipos; una masa celular interna llamada embrioblasto y una capa celular externa denominada trofoblasto (Moore et al., 2016).

La zona pelúcida (ZP) se pierde después del ingreso del embrión a la cavidad uterina y previa a la implantación (Carlson; Arteaga et al.; Moore et al., 2016), proceso mediante el cual el blastocito se adhiere al epitelio endometrial, lo que ocurre aproximadamente 6 a 7 días post fecundación, y en donde el trofoblasto del polo embrionario comienza a adherirse firmemente al epitelio del endometrio, para luego sumergirse en el estroma endometrial (Carlson; Webster et al, 2013). En condiciones normales, el blastocisto se implanta en el endometrio, en la parte superior de las paredes anterior o posterior del cuerpo del útero (Arteaga et al.; Schoenwolf et al., 2015). El éxito del proceso de implantación depende de un alto grado de preparación y coordinación entre el embrión y el endometrio. Éste último responde en forma endocrina e inmunitaria, facilitando el "reconocimiento materno de la gestación", e impide que el cuerpo de la madre rechace al embrión y le permita su nidación, crecimiento y desarrollo (Gratacos et al.; Carlson; Webster et al., 2013; Moore et al., 2016). En la implantación, la mucosa del útero se encuentra en la fase secretora y previamente, el epitelio uterino ha segregado citoquinas y quimioquinas en el flujo uterino. Paralelamente a ello, en la superficie del trofoblasto aparecen receptores para estas citoquinas. Posteriormente el trofoblasto experimenta una rápida proliferación y diferenciación dando origen a dos tipos celulares denominados: citotrofoblasto y sinciotrofoblasto (Carlson; Pérez Sánchez \& Donoso, 2011; Webster \& de Wreede, 2013; Moore et al., 2016).

Por otra parte, las células trofoblásticas del polo embrionario del embrión interaccionan con el epitelio de la mucosa uterina, donde participan integrinas expresadas por el trofoblasto, y las moléculas de la matriz extracelular, laminina y fibronectina. Los receptores de integrina para laminina promueven la adhesión, mientras que aquellos para fibronectina estimulan la migración. Estas moléculas interaccionan a lo largo de vías de transducción de señales para regular la diferenciación del trofoblasto (Gómez, 2003; Arteaga et al.).

Al final de la primera semana el blastocito se encuentra implantado superficialmente en la capa compacta del endometrio, debido a la capacidad invasiva del sinciotrofoblasto, el cual secreta enzimas que erosionan el tejido materno (Gómez; Carlson).

La implantación del blastocito continúa durante la segunda semana por acción de las enzimas proteolíticas producidas por el sinciotrofoblasto, las prostaglandinas derivadas de COX-2 presentes en el lugar de la implantación y por la adaptación del endometrio ("reacción decidual"). Las células adyacentes al sitio de la implantación, responden a la presencia del embrión y a la progesterona secretada por el cuerpo lúteo, siendo éstas metabólicamente activas y secretoras, denominándose células deciduales (Schoenwolf et al.). Las glándulas endometriales cercanas a la implantación, se alargan y la pared uterina local se torna altamente vascularizada y edematosa. Las secreciones de las células deciduales y glándulas endometriales podrían incluir factores de crecimiento y metabolitos que mantendrían el crecimiento del embrión implantado (Schoenwolf et al.; Carlson). Por otra parte, una hormona de gran importancia secretada por el sinciotrofoblasto es la Gonadotropina Coriónica Humana (hCG), que cumple la función de mantener la actividad del cuerpo lúteo, y con ello el desarrollo del endometrio, a través de la secreción de progesterona. (Moore et al., 2016; Eynart et al., 2011).

El objetivo de esta revisión bibliográfica es complementar lo descrito en la literatura clásica de embriología humana sobre interacción de endometrio-blastocito. 


\section{MATERIAL Y MÉTODO}

La búsqueda bibliográfica en línea se llevó a cabo en la base de datos MEDLINE usando los términos en inglés "implantation", "endometrium" y "embryo". También se realizó una búsqueda manual, en la cual se incluyeron artículos de revistas no indexadas, libros de texto y atlas. La última búsqueda se llevó a cabo el día 31 de octubre de 2017.

Se consideraron criterios de inclusión y exclusión para la selección de los artículos y otros recursos bibliográficos. Entre los criterios de inclusión se consideraron estudios relizados en humanos, artículos de revisión y experimentación, publicados en los últimos 5 años. Como criterios de exclusión se consideraron artículos que utilizaran animales, estudios sobre fertilidad in vitro, patologías asociadas y artículos no relacionados al tema. No se establecieron restricciones de idioma, sin embargo, todos los artículos seleccionados son de lengua inglesa, basándose la selección inicial en el análisis del título y resumen de los mismos; aquellos artículos duplicados y que no cumplieron con los criterios establecidos fueron eliminados. Una vez completada la selección, se examinaron los textos completos, en los cuales se aplicaron nuevamente los criterios de exclusión.

\section{RESULTADOS Y DISCUSIÓN}

La búsqueda arrojó un total de 560 artículos, cuyo análisis de los títulos y resúmenes resultó en 475 trabajos excluidos, a partir de los diferentes criterios de exclusión antes descritos. Por lo tanto, se obtuvieron 85 artículos, en

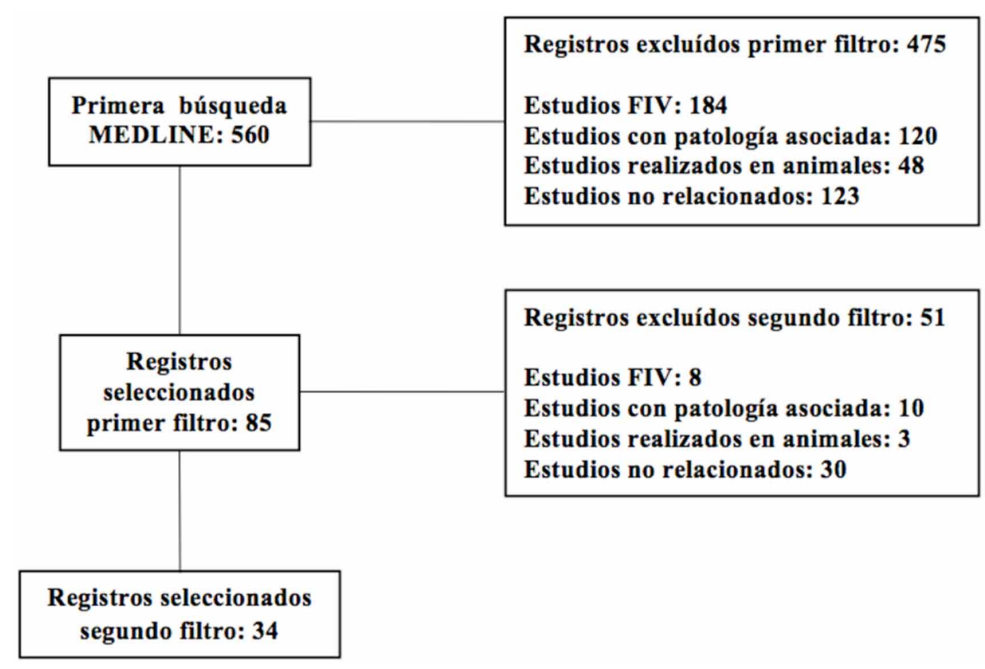

Fig. 1. Diagrama de flujo de la búsqueda bibliográfica. los cuales se realizó el análisis del texto completo. De estos artículos, luego de la aplicación de los criterios de exclusión, se obtuvieron un total de 34 estudios (Fig. 1).

Endometrio. El endometrio es la porción más interna del útero, compuesto por una capa funcional y una capa basal. La capa funcional, morfológicamente, está constituida por el epitelio luminal, el glandular, el estroma y los compartimentos vasculares. Funcionalmente es la encargada de la proliferación, secreción y degeneración tisular, mientras que la capa basal le confiere al endometrio la capacidad regenerativa. El endometrio sufre modificaciones durante el ciclo menstrual determinando las respectivas fases: proliferativa, secretora y menstrual. Este ciclo, también llamado endometrial, es regulado por esteroides ováricos y por células vecinas a través de señales paracrinas (Ruiz-Alonso et al., 2012). Cada una de estas fases expresa distintos genes que pueden ser caracterizados mediante un perfil genómico, que se denomina transcriptomía genómica (Garrido-Gómez et al., 2013). Se han descrito diferencias proteicas significativas entre las distintas fases del ciclo y su correlación con la receptividad endometrial (Chen et al., 2015).

La fase secretora del ciclo endometrial se sitúa entre la ovulación y la menstruación, se reconoce como la fase donde el endometrio adopta un fenotipo receptivo que permite la implantación del blastocisto (Ruiz-Alonso et al.). En esta fase se lleva a cabo el proceso denominado decidualización, que corresponde a una transformación morfológica y funcional del estroma, necesaria para el control de la invasión embrionaria, durante la cual los fibroblastos del estroma endometrial se transforman en células deciduales, proporcionado una matriz nutritiva esencial para la implantación. Este cambio morfológico es en parte mediado por quinasas ligadas a integrinas (ILK), las cuales participan en la transformación morfológica de las células del estroma endometrial (ESC) a través de la organización del citoesqueleto (Yen et al., 2017).

Sin embargo, no solo quinasas intervienen en este complejo proceso morfofuncional, sino que son variados los agentes morfógenos, proteicos, moleculares y hormonales que participan en la decidualización. Dentro de los morfógenos involucrados durante la fase secretora y de decidualización endometrial se decribe recientemente la participación de Nodal (morfógeno de la superfamilia del factor de crecimiento transformante b), con un rol dinámico en el ciclo endometrial. LEFTYA es otro morfógeno, descrito como antagónico a Nodal, que actúa como mediador de enzimas 
proteolíticas denominadas metaloproteinasas (MMP1, MMP3 y MMP9), que se relacionan con la degradación de la función uterina. De esta manera ambosmorfógenos se ven expresados a lo largo del ciclo endometrial en distintas concentraciones, con participación antagónicas durante este proceso (Park \& Dufort, 2013).

Los agentes proteicos han sido ampliamente estudiados, pudiéndose observar mayores niveles de expresión de proteínas de adhesión, fundamentales para el proceso de implantación, entre las que se encuentra la periostina, que ha sido identificada en células deciduales en mujeres embarazadas (Morelli, Misaggi et al. 2014). Asimismo, una proteína sensible al calcio e implicada en la transducción de señales, la $\mathrm{S} 100 \mathrm{P}$, se expresa en altas concentraciones durante la fase secretora, influenciada por acción de la progesterona (Zhang et al., 2012). Otras proteínas descritas corresponden a las acuaporinas AQP 1 y 2, sitúadas en las células endoteliales y epiteliales, respectivamente, donde forman poros en las membranas para el transporte de agua. La AQP 1 regula la formación del edema estromal, mientras que la AQP 2 participa en el intercambio de agua entre el lumen y el estroma endometrial, por lo que su sobreexpresión se asocia con fallas de la implantación (Zhou et al., 2013). Otros estudios describen moléculas cuya baja expresión o mutación genética ocasionaría una falla en el proceso de implantación, como es el caso del factor inhibitorio de la leucemia, que posee la capacidad de inducir la expresión de integrinas al ser estimulado por el ácido benzoico (Choi et al., 2017). En este contexto, moléculas como la Forkhead Box M1 (FoxM1) (anteriormente conocida como HNF-3), un factor transcripcional, desempeña un papel crucial en la proliferación celular y la progresión del ciclo celular. El FoxM1 se expresa dinámicamente en el endometrio humano durante el ciclo menstrual y se ha demostrado que FoxM1 inhibe de manera parcial la expresión de ciclina B1, retrasando la transición de fase G2 / M del ciclo celular, durante la proliferación de las células del estroma endometrial humano (HESCs). Además, la pérdida de expresión de FoxM1 bloquea la diferenciación de HESCs en respuesta a estrógeno, progesterona y AMPc (Jiang et al., 2015).

El AMPc, nucleótido y segundo mensajero de gran importancia, es una de las moléculas más estudiadas por su participación en el proceso de decidualización, en conjunto con la enzima Proteína Quinasa A (PKA), que depende de la expresión del primero para su acción. Así, la expresión de AMPc y su interacción AMPc / PKA, al igual que los mecanismos moleculares subyacentes a la decidualización, son de interés en el ámbito de la investigación. En este contexto, estudios recientes indican la presencia de una fosfolipasa para la diferenciación de las HESCs a través de la inducción y expresión de AMPc (Lee et al., 2016). Esta fosfolipasa corresponde a la PLD 1 (fosfolipasa D1), que al inducir al ácido fosfatídico (PA), genera cambios morfológicos en las HESCs, junto con niveles aumentados de marcadores de decidualización y de trascripción, como IGFP1 y prolactina, respectivamente. Sin embargo, los mecanismos moleculares específicos por el cual el ácido fosfatídico podría regular la decidualización son aún desconocidos. No obstante, se ha propuesto que uno de estos mecanismos corresponde al estímulo de unión PKB-PP2A por parte del ácido fosfatídico; PP2A (fosfoproteína fosfatasa 2 A), induce la fosforilación de PKB (Proteína Kinasa B) inactivándolo, lo que genera la expresión de Fox01, que es el responsable de la activación de los genes relacionados con la decidualización (Lee et al.).

En este sentido, existe un agente que se señala como uno de los controladores más críticos en la diferenciación de HESCs, generando un gran interés por sus múltiples vías descendentes que desencadena. Este corresponde al factor de transcripción $\mathrm{C} / \mathrm{EBPb}$, que regula muchos procesos de la decidualización, tal como la expresión de ciclina E (con ello el complejo E-cdk2), que a su vez se relaciona con la regulación del ciclo celular del tejido endometrial, y consecuentemente en la intervención de la diferenciación de estas células. Asimismo, regula la expresión de prolactina decidual, la cual se asocia al proceso de diferenciación de las células estromales. Por otra parte, interviene en la vía BMP2-WNT4, que se relaciona con la formación y función del tejido decidual. Otra interacción de $\mathrm{C} / \mathrm{EBPb}$ es su asociación con proteínas como la STAT3, la cual es una mediadora de citoquinas como la IL-11, correspondiente a una vía de señal de citocina involucrada en la decidualización, lo que se ha relacionado con el establecimiento de una gestación exitosa. Es decir, $\mathrm{C} / \mathrm{EBPb}$ es un regulador crítico de la proliferación y diferenciación de las células del estroma endometrial humano (Wang et al., 2012).

Por otra aparte, los factores hormonales presentes en el proceso de decidualización endometrial corresponden principalmente a la progesterona y a la gonadotropina coriónica humana (hCG). Ésta última es producida por el trofoblasto, con acción directa en el endometrio y contribuye a que éste adquiera un fenotipo receptivo. Una molecula estudiada es la galectin-3, que podría estar regulada por hCG en la preparación del endometrio, participando en procesos celulares de proliferación y apoptosis, necesarios para una exitosa implantación (Yang et al., 2013)

Interacción endometrio trofoblasto. La implantación comienza durante un periodo de tiempo denominado "ventana de implantación" que transcurre en el período de la fase de secretora, donde el endometrio se vuelve receptivo, lo que permite la adhesión del embrión. Esta receptividad puede durar entre 12 horas y 2 días (Franasiak et al., 2016). 
Cuando el endometrio se encuentra en fase receptiva expresa moléculas de adhesión como mucinas, cadherinas e integrinas. Además posee receptores para colágeno, laminina, fibronectina, ácido hialurónico y heparán sulfato; que se uniran a las integrinas del trofoblasto (Gómez). Las integrina secretan hCG, la cual favorece la secreción de progesterona desde el cuerpo lúteo, procesos que facilitan la interacción entre el trofoblasto y el epitelio endometrial. Esta interacción se desarrolla a través de las siguientes etapas: aposición, adhesión, migración e invasión, donde participan distintos factores moleculares y hormonales que regula cada uno de ellas (Moore et al., 2009; Cuman et al., 2014).

Aposición. La aposición es un proceso dinámico durante el cual el blastocisto, que flota libremente en el útero, y el endometrio receptivo comienzan a interactuar (Gnainsky et al., 2014). Es aquí donde se describen citocinas como las quimiocinas, que actúan como quimioatrayentes, siendo las primeras en aparecer durante la aposición, entre las que destaca es la IP-10, observadando la expresión de receptores para IP-10 en el trofoectodermo, como el CXCR3 (Sela et al., 2013). Además, se ha descrito a la familia de IL-6, un tipo de citoquinas, que participan en las primeras etapas de la implantación, la IL-11 y LIF que estimulan a las moléculas de adhesión, creando gradientes que atraen al blastocisto al lugar de implantación (Van Sinderen et al., 2013).

Otros agentes que participan en esta interacción blastocisto-endometrio son los exosomas, que corresponden a pequeñas vesículas con contenido heterogéneo y que poseen una carga exosómica específica, que es regulada hormonalmente por estrógenos y progesterona, y al aproximarse a las células del trofoblasto, favorecen la capacidad adhesiva para iniciar una implantación exitosa. Estos exosomas no solo intervienen en la presente etapa de interacción, sino que su acción se extiende a etapas posteriores, tal como se describe más adelante (Greening et al., 2016).

Así como existe las señales del endometrio hacia el blastocisto, existen mediadores que provienen del trofoblasto y que generan cambios en el endometrio. En este sentido, aparece el PDGF-AA (Factor de crecimiento derivado de plaquetas AA), el que constituye una señal producida por el trofoblasto cuya acción es la atracción de células estromales al sitio de implantación. Sumado a esta interaccion de señales, existen factores locales que cumplen una función similar, reforzando la migración de las células deciduales del estroma de manera aleatoria (quimiokinesis) o dirigida (quimiotaxis), que corresponden a PDGF-BB y HB-EGF. En conclusión, la mediación de estos factores tiene como acción final establecer procesos de remodelación necesa- rios para el establecimiento y mantenimiento de la gestación (Schwenke et al., 2013).

Adhesión. En esta etapa se expresan las integrinas, las cuales son intermediarias entre el endometrio y el blastocisto facilitando la fijación del trofoblasto o trofoectodermo, con las células epiteliales del endometrio en la capa compacta (López Serna, 2012).

En esta etapa la interacción blastocisto-endometrio se realiza mediante moléculas de adhesión tales como troponina, cadherinas e integrinas, asociadas a varios ligandos que contienen fibronectina, vitronectina, trombospondina y osteopontina (Gnainsky et al.). Notch, una proteína transmembrana de señalizacion se describe en las interacciones endometrio-trofoblasto durante las etapas de adhesión e invasión (Cuman et al.); también Galectin-7, se ha encontrado en las células epiteliales endometriales y en el sincitiotrofoblasto del blastocisto; su expresión se encuentra anormalmente elevada en el endometrio de mujeres con falla de implantación, sugiriendo que la Galectin-7 facilita la adhesión del embrión al endometrio, pero una expresión elevada de ésta puede dar como resultado una adhesión anormal (Menkhorst et al., 2014).

Tal como se señaló en la etapa de aposición, los exosomas hacen su aparición en etapas más avanzadas de la interacción, donde se señala que el contenido de estas vesículas tiene lugar en el proceso de adhesión. Algunas de las proteínas presentes como carga del exosoma son la Fibulina1, que es una glicoproteína asociada con la remodelación de la matriz extracelular, adhesión celular y la interacción de la membrana basal, por lo que podría ser una molécula clave implicada en la implantación embrionaria. Del mismo modo, como parte del contenido exosomal aparece la proteína HSPG2 que cumple la función de facilitar la adhesión del trofoblasto. Asimismo, la Galectin-1 se asocia a la evasión inmune del trofoblasto, mediante la regulación del antígeno $\mathrm{G}$ de los leucocitos. De esta forma, los exosomas a través de su contenido intervienen en el proceso de adhesión en el endometrio, aunque también inducen cambios en el blastocisto. Inclusive, son capaces de regular parcialmente la adherencia de células a través de la señalización de adhesión FAK (focal quinasa activa) (Greening et al.).

Migración-Invasión. La invasión es el paso final de la implantación, durante el cual el trofoblasto rompe el revestimiento epitelial del útero y penetra en el estroma endometrial (Gnainsky et al.), proceso regulado en espacio y tiempo. Después de la unión del epitelio endometrial al trofoblasto, éste altera e invade la decidua hasta el tercio proximal del miometrio. Se sabe que un mecanismo para la destrucción de las células epiteliales es la apoptosis, durante la invasión 
del trofoblasto. La Sdc-1 (Syndecan-1) miembro de la familia de proteoglicanos de heparan sulfato (HS) que se encuentra en la superficie celular, cumple un papel fundamental en la interacción blastocisto-endometrio, especialmente en el proceso de apoptosis. La Sdc1 parece ser un factor importante que influye en la profundidad de la invasión, mediante la regulación de la apoptosis en las células endometriales maternas (Boeddeker et al., 2014). Por otra parte, los Sdcs se expresan en la superficie de células adherentes y no adherentes, sirviendo como correceptores para diversos ligandos, como factores de crecimiento, proteasas, inhibidores de proteasa, quimiocinas y citoquinas que intervienen en múltiples procesos fisiológicos y/o patológicos, incluída la regulación de proteínas del citoesqueleto. Todo esto le otorga un papel importante en la invasión celular, por lo que Sdc-1 podría actuar como un marcador de diagnóstico para la falla o trastorno de la implantación (Baston-Buest et al., 2017).

En este proceso de invasión, es crítica la participación de enzimas que degradan la membrana basal. Aquí aparecen las metaloproteinasas, entre las que destacan la MMP2 y MMP-9, la primera es la principal reguladora de la invasión del trofoblasto al inicio del embarazo. Sin embargo, debe haber un equilibrio en el proceso enzimático de degradación, especialmente en la protección y mantención de la integridad de los vasos sanguíneos, lo cual está regulado por inhibidores de las MMP, denominados TIMP-1 y TIMP2. Por tanto, existe una conformación de un complejo MMP9 y MMP-2 / TIMP-2, el cual está involucrado en la remodelación del tejido intrauterino durante la invasión (Nissi et al., 2013).

Uno de los factores mencionados en la decidualizacion, que también actúa en el proceso de invasión, es el LIF (Factor Inhibidor de Leucemia), que interviene a través de varios mecanismos en esta etapa, estimulando las células del trofoblasto, las que adquieren la capacidad de invadir la decidua, iniciando la implantación. El LIF también estimula la invasión del trofoblasto y su proliferación mediante cascadas de señalización de STAT3 y STAT1, que tienen como consecuencia la estimulación de la adhesión a los elementos de la matriz extracelular, como es la fibronectina, vitronectina y laminina. Por otro lado, también se señala que regula negativamente la expresión de genes que codifican TIMP1, TIMP2 y TIMP3, antes descrito, son inhibidores de las metaloproteinasas, ayudando a reducir la expresión de éstos y de esta forma potenciar la invasión trofoblastica (Salleh \& Giribabu, 2014).

Como es sabido, el éxito de la implantación y la placentación del embrión dependen de la invasión adecuada del trofoblasto en el estroma endometrial materno. La gonadotropina coriónica humana (hCG) es una de las primeras señales secretadas, que al derivar de embriones en las células mononucleares de sangre periférica (PBMC), expresan abundantemente los receptores de hCG. La hCG podría activar PBMC para inducir la secreción de varias citoquinas que promueven la invasión embrionaria, sugiriendo un ciclo de retroalimentación positiva entre ambas. Asimismo, estudios recientes sugieren la utilización de PBMC surgidas por el mecanismo descrito, para terapias en pacientes con falla de implantación (Yu et al., 2015).

Otra de las múltiples vías por la que interviene la hCG, es la modulación de la receptividad de células endometriales a IL-1. La hCG y la IL-1 actúan en conjunto para la modulación de las funciones angiogénicas, inmunitarias y de remodelación tisular de las células del estroma endometrial para favorecer la invasión del trofoblasto. Se sugiere que el mecanismo consiste en la estimulación sinérgica de la Quimiocina CC Motivo ligando (CCL2) y el factor de crecimiento endotelial C (VEGFC), y la inhibición de TIMP3 moderando la acción de MMP9 activada por IL1B, induciendo de manera efectiva la invasión (Bourdiec et al., 2013).

Por último, se ha descrito que la hCG secretada por el embrión, modula la respuesta inmune en el endometrio. Ya que esta hormona regula el aumento de C3 (proteína central del complemento) en zonas específicas del endometrio, así como en otras zonas impide su sobre acción. Todo esto trae como consecuencia que la hCG module la interacción del embrión con el endometrio, por medio de la regulación del sistema inmune (Palomino et al., 2013). En este sentido, el control adecuado por parte de inmunomoduladores para evitar una reacción negativa del endometrio ante el embrión y su invasión, resulta trascendental para el éxito de la implantación. En este contexto, se describe un péptido denominado péptido intestina vasoactivo (VIP), el cual contribuye a la inducción de un microambiente tolerogénico materno fisiológico, que interviene como un factor que modula las respuestas efectoras Th1 / Treg (células T reguladoras especializadas). Además, interviene en la producción de mediadores pro/antiinflamatorios que promueven un equilibrio general, favoreciendo la tolerancia hacia los antígenos de trofoblasto (Fraccaroli et al., 2012).

Si se indaga aún más en el aspecto inmunológico necesario para la modulación de una implantación exitosa, en la actualidad es conocido el papel importante que juega la hormona progesterona. Aun cuando el mecanismo de acción de inmunomodulacion es incierto, se señala que ejerce un efecto antiinflamatorio durante la implantación por medio de la supresión de la entrada de neutrófilos y macrófagos inducidas por estradiol. Se propone además, la participa- 
ción de co-moduladores como HOXA10, cuya función es actuar como medidor en la acción inmunomoduladora de la progesterona. Esto se basa en que la deficiencia de HOXA10, se asocia con una alteración inmunológica severa durante la implantación (Bhurke et al., 2016).

Dentro del proceso de invasión del trofoblasto en la decidua, se destaca el comportamiento móvil de las células estromales endometriales deciduales (CEED). Éstas se consideran de importancia crítica para la implantación embrionaria y la programación de la gestación humana, debido a que la regulación de la motilidad de las CEED puede ser un mecanismo importante en el proceso normal de implantación embrionaria. Esta acción de la motilidad está promovida por la hormona liberadora de gonadotropina tipo II (GnRH-II) que es un agonista de las CEED. La GnRH-II tiene además efectos significativos sobre la invasión y migración de las CEED, ya que provocaría la estimulación directa de dichos procesos mediante el aumento de la expresión y actividad proteolítica de MMP-2 y MMP-9, quienes degradan de forma específica a la membrana basal, validando la inducción de la motilidad de CEED por parte de la GnRH-II (Wu et al., 2015).

La invasión también involucra modificaciones a nivel vascular. Donde la enzima CORIN, a través de su mecanismo de acción promueve la invasión de trofoblasto en las arterias espirales mediante la activación de pro-ANP a ANP (péptido natriurético auricular), el cual es un péptido de señalización conocido por su papel en la regulación de la presión arterial (Kaitu'u-Lino et al., 2013).

Adicionalmente, se describe la participación de mediadores de la inflamación, ya que el proceso de invasión tiene un componente inflamatorio involucrado. Entre los agentes aparece el factor de crecimiento insulínico, específicamente IGF-2 en conjunto con sus moduladores, cuya acción es la regulación de la invasión. Se describe además, que la familia de las interleucinas (IL), en especial la IL-1b regula las proteínas remodeladoras de la matriz extracelular (Van Sinderen et al.).

En los últimos años han emergido nuevos actores importantes en el proceso de la implantación. Uno de ellos corresponde a los canales iónicos. Se describe que existen más de 14 tipos de canales iónicos que se expresan en el endometrio o células de origen endometrial. Lo más relevante en el presente proceso de invasión, es la participación de varios canales iónicos en la regulación de la expresión de genes asociados con la implantación. Se señala además, que la expresión anormal de canales iónicos se asocia de manera importante con la falla de implantación (Ruan et al., 2014).

\section{CONCLUSIÓN}

Durante la implantación humana se genera una interacción entre el endometrio y el trofoblasto, con la participación de moléculas reguladoras de proliferación y diferenciación. Entre éstas se describen diversos factores hormonales, moleculares y de expresión génica, tales como: proteínas de adhesión, factores inhibitorios, factores transcripcionales, factores de crecimiento, que participan en el proceso de implantación. Sin embargo, los mecanismos específicos de acción e interacción de éstos requieren aún ser investigados, con el propósito de responder a las interrogantes de los investigadores, en la enseñanza e investigación, para contribuir finalmente al manejo clínico de la mujer durante su ciclo reproductivo.

PRIETO, G. R.; VIDAL, S. N. E.; LIZAMA, P. R.A.; MIRANDA, K. R. E.; NAHUELCURA, M. N. E.; ORTEGA, S. M. E.; OTTONE, N. E. \& DEPPE, A. M. Endometrium-trophoblast interaction in human implantation: Review of literature. Int. J. Morphol., 37(2):397-405, 2019.

SUMMARY: A successful pregnancy requires a series of interactions, mediated by hormonal, molecular and immunomodulation phenomena. One of these interactions is between the endometrium and the blastocyst, before and during the implantation process. The objective of this literature review is to complement what is described in the classic human embryology literature on endometrial-blastocyst interaction. The bibliographic search was carried out in the MEDLINE database using the terms "implantation", "endometrium" and "embryo", and a manual search was carried out, which included articles from non-indexed journals, textbooks and atlases. Inclusion and exclusion criteria were considered for the selection of articles and other bibliographic resources, including human studies, review and experimentation articles, published in the last 5 years. Articles with animals as experimental subjects, in vitro fertility studies, associated pathologies and articles not related to the subject were excluded. When the selection was completed, the complete texts were examined, in which the exclusion criteria were applied again The search yielded a total of 560 articles, whose analysis of titles and abstracts resulted in 475 excluded works, in relation to different exclusion criteria described above. Therefore, 85 articles were obtained, in which the complete text analysis was performed. From these articles, a total of 34 studies were obtained and the contents selected in this review were: Endometrium, Endometrium trophoblast, Aposition, Adhesion and Migration-Invasion. During the implantation, aninteraction between the endometrium and the trophoblast is generated, with the participation of regulatory molecules of proliferation and differentiation, such as hormonal, molecular and gene expression factors. However, the specific mechanisms of action and interaction must continue to be investigated, to answer questions in the field of human growth and development.

KEY WORDS: Implantation; Endometrium; Trophoblast. 


\section{REFERENCIAS BIBLIOGRÁFICAS}

Arteaga, M. \& García, M. I. Embriología Humana y Biología del Desarrollo. Ciudad de México, Editorial Panamericana, 2013.

Baston-Buest, D. M.; Altergot-Ahmad, O.; Pour, S. J.; Krüssel, J. S.; Markert, U. R.; Fehm, T. N. \& Bielfeld, A. P. Syndecan-1 acts as an important regulator of CXCL1 expression and cellular interaction of human endometrial stromal and trophoblast cells. Mediators Inflamm., 2017:8379256, 2017.

Bhurke, A. S.; Bagchi, I. C. \& Bagchi, M. K. Progesterone-regulated endometrial factors controlling implantation. Am. J. Reprod. Immunol., 75(3):237-45, 2016.

Boeddeker, S. J.; Baston-Buest, D. M.; Altergot-Ahmad, O.; Kruessel, J. S. \& Hess, A. P. Syndecan-1 knockdown in endometrial epithelial cells alters their apoptotic protein profile and enhances the inducibility of apoptosis. Mol. Hum. Reprod., 20(6):567-78, 2014.

Bourdiec, A.; Calvo, E.; Rao, C. V. \& Akoum, A. Transcriptome analysis reveals new insights into the modulation of endometrial stromal cell receptive phenotype by embryo-derived signals interleukin-1 and human chorionic gonadotropin: possible involvement in early embryo implantation. PLoS One, 8(5):e64829, 2013.

Carlson, B. M. Embriología Humana y Biología del Desarrollo. $4^{\mathrm{a}} \mathrm{ed}$. Barcelona, Elsevier, 2009.

Cuman, C.; Menkhorst, E.; Winship, A.; Van Sinderen, M.; Osianlis, T.; Rombauts, L. J. \& Dimitriadis, E. Fetal-maternal communication: the role of Notch signalling in embryo implantation. Reproduction, 147(3):R75-86, 2014.

Chen, Q.; Zhang, A.; Yu, F.; Gao, J.; Liu,Y.; Yu, C.; Zhou, H. \& Xu, C. Label-free proteomics uncovers energy metabolism and focal adhesion regulations responsive for endometrium receptivity. $J$. Proteome Res., 14(4):1831-42,2015.

Choi, H. J.; Chung, T. W.; Park, M. J.; Kim, H. S.; You, S.; Lee, M. S.; Joo, B. S.; Lee, K. S.; Kim, K. J.; Wee, G.; Kim, C. Y.; Kim, C. H. \& Ha, K. T. Benzoic acid enhances embryo implantation through LIFdependent expression of integrin aVb3 and aVb5. J. Microbiol. Biotechnol., 27(4):668-77, 2017.

Eynard, A. R.; Valentich, M. A. \& Rovasio, R. A. Histología y Embriología del Ser Humano. Bases Moleculares. $4^{\mathrm{a}}$ ed. Buenos Aires, Médica Panamericana, 2011.

Fraccaroli, L.; Grasso, E.; Hauk, V.; Cortelezzi, M.; Calo, G.; Pérez Leirós, C. \& Ramhorst, R. Defects in the vasoactive intestinal peptide (VIP)/ VPAC system during early stages of the placental-maternal leucocyte interaction impair the maternal tolerogenic response. Clin. Exp. Immunol., 170(3):310-20, 2012.

Franasiak, J. M.; Ruiz-Alonso, M.; Scott, R. T. \& Simón, C. Both slowly developing embryos and a variable pace of luteal endometrial progression may conspire to prevent normal birth in spite of a capable embryo. Fertil. Steril., 105(4):861-6, 2016.

Garrido-Gómez, T.; Ruiz-Alonso, M.; Blesa, D.; Diaz-Gimeno, P.; Vilella, F. \& Simón, C. Profiling the gene signature of endometrial receptivity: clinical results. Fertil. Steril., 99(4):1078-85, 2013.

Gnainsky, Y.; Dekel, N. \& Granot, I. Implantation: mutual activity of sex steroid hormones and the immune system guarantee the maternalembryo interaction. Semin. Reprod. Med., 32(5):337-45, 2014.

Gómez, C. Embriología Humana. Atlas y Texto. Buenos Aires, Ateneo, 2003.

Gratacos, E.; Gómez, R.; Romero, R.; Nicolaieds, K \& Cabero, L. Medicina Fetal. Madrid, Médica Panamericana, 2008.

Greening, D. W.; Nguyen, H. P.; Elgass, K.; Simpson, R. J. \& Salamonsen, L. A. Human endometrial exosomes contain hormonespecific cargo modulating trophoblast adhesive capacity: Insights into endometrial-embryo interactions. Biol. Reprod., 94(2):38, 2016.
Jiang, Y.; Liao, Y.; He, H.; Xin, Q.; Tu, Z.; Kong, S.; Cui, T.; Wang, B.; Quan, S.; Li, B.; Zhang, S. \& Wang, H. FoxM1 directs STAT3 expression essential for human endometrial stromal decidualization. Sci. Rep., 5:13735, 2015.

Kaitu'u-Lino, T. J.; Ye, L.; Tuohey, L.; Dimitriadis, E.; Bulmer, J.; Rogers, P.; Menkhorst, E.; Van Sinderen, M.; Girling, J. E.; Hannan, N. \& Tong, S. Corin, an enzyme with a putative role in spiral artery remodeling, is up-regulated in late secretory endometrium and first trimester decidua. Hum. Reprod., 28(5):1172-80, 2013.

Lee, S. Y.; Lee, Y. Y.; Choi, J. S.; Yoon, M. S. \& Han, J. S. Phosphatidic acid induces decidualization by stimulating Akt-PP2A binding in human endometrial stromal cells. F. E. B. S. J., 283(22):4163-75, 2016.

López Serna, N. Biología del Desarrollo. Cuaderno de Trabajo. Libro en Internet. McGraw-Hill Interamericana Editores, 2012. Disponible en: https://accessmedicina.mhmedical.com/ book.aspx?bookID=1476

Menkhorst, E. M.; Gamage, T.; Cuman, C.; Kaituu-Lino, T. J.; Tong, S. \& Dimitriadis, E. Galectin-7 acts as an adhesion molecule during implantation and increased expression is associated with miscarriage. Placenta, 35(3):195-201, 2014

Moore, K. L.; Persaud, T. V. N. \& Torchia, M. G. Embriología Clínica. 10a ed. Barcelona, Elsevier, 2016.

Moore, K. L.; Persaud, T. V. N. \& Torchia, M. G. The Developing Human: Clinically Oriented Embryology. $9^{\text {a }}$ ed. Philadelphia, Saunders/Elsevier, 2009.

Nissi, R.; Talvensaari-Mattila, A.; Kotila, V.; Niinimäki, M.; Järvelä, I. \& Turpeenniemi-Hujanen, T. Circulating matrix metalloproteinase MMP-9 and MMP-2/TIMP-2 complex are associated with spontaneous early pregnancy failure. Reprod. Biol. Endocrinol., 11:2, 2013.

Palomino, W. A.; Argandoña, F.; Azúa, R.; Kohen, P. \& Devoto, L. Complement $\mathrm{C} 3$ and decay-accelerating factor expression levels are modulated by human chorionic gonadotropin in endometrial compartments during the implantation window. Reprod. Sci., 20(9):1103-10, 2013.

Park, C. B. \& Dufort, D. NODAL signaling components regulate essential events in the establishment of pregnancy. Reproduction, 145(2):R55-64, 2013

Pérez Sánchez, A. \& Donoso, E. Obstetricia. $4^{\mathrm{a}}$ ed. Santiago de Chile, Mediterráneo, 2011.

Ruan, Y. C.; Chen, H. \& Chan, H. C. Ion channels in the endometrium regulation of endometrial receptivity and embryo implantation. Hum. Reprod. Update, 20(4):517-29, 2014.

Ruiz-Alonso, M.; Blesa, D. \& Simón, C. The genomics of the human endometrium. Biochim. Biophys. Acta, 1822(12):1931-42, 2012.

Sadler, T. W. Langman. Embriología Médica. 12a. ed. Barcelona, Lippincott Williams \& Wilkins, 2012.

Salleh, N. \& Giribabu, N. Leukemia inhibitory factor: roles in embryo implantation and in nonhormonal contraception. Scientific World Journal, 2014:201514, 2014.

Schoenwolf, G. C.; Bleyl, S. B.; Brauer, P. R. \& Francis-West, P. H. Larsen's Human Embryology. Philadelphia, Elsevier/Churchill Livingstone, 2015.

Schwenke, M.; Knöfler, M.; Velicky, P.; Weimar, C. H.; Kruse, M.; Samalecos, A.; Wolf, A.; Macklon, N. S.; Bamberger, A. M. \& Gellersen, B. Control of human endometrial stromal cell motility by PDGF-BB, HB-EGF and trophoblast-secreted factors. PLoS One, 8(1):e54336, 2013

Sela, H. Y.; Goldman-Wohl, D. S.; Haimov-Kochman, R.; Greenfield, C.; Natanson-Yaron, S.; Hamani, Y.; Revel, A.; Lavy, Y.; Singer, O.; Yachimovich-Cohen, N.; Turetsky, T.; Mandelboim, O.; Reubinoff, B. \& Yagel, S. Human trophectoderm apposition is regulated by interferon g-induced protein 10 (IP-10) during early implantation. Placenta, 34(3):222-30, 2013. 
PRIETO, G. R.; VIDAL, S. N. E.; LIZAMA, P. R. A.; MIRANDA, K. R. E.; NAHUELCURA, M. N. E.; ORTEGA, S. M. E.; OTTONE, N. E. \& DEPPE, A. M. Interacción endometrio trofoblasto, en la implantación humana: Revisión de la literatura. Int. J. Morphol., 37(2):397-405, 2019.

Van Sinderen, M.; Menkhorst, E.; Winship, A.; Cuman, C. \& Dimitriadis, E. Preimplantation human blastocyst-endometrial interactions: the role of inflammatory mediators. Am. J. Reprod. Immunol., 69(5):427-40, 2013.

Wang, W.; Taylor, R. N.; Bagchi, I. C. \& Bagchi, M. K. Regulation of human endometrial stromal proliferation and differentiation by $\mathrm{C} /$ $\mathrm{EBPb}$ involves cyclin E-cdk2 and STAT3. Mol. Endocrinol., 26(12):2016-30, 2012.

Webster, S. \& de Wreede, R. Embriología. Lo Esencial de un Vistazo. Madrid, Médica Panamericana, 2013.

Wu, H. M.; Huang, H. Y.; Lee, C. L.; Soong, Y. K.; Leung, P. C. \& Wang, H. S. Gonadotropin-releasing hormone type II (GnRH-II) agonist regulates the motility of human decidual endometrial stromal cells: possible effect on embryo implantation and pregnancy. Biol. Reprod., 92(4):98, 2015

Yang, H.; Lei, C. X. \& Zhang, W. Human chorionic gonadotropin (hCG) regulation of galectin-3 expression in endometrial epithelial cells and endometrial stromal cells. Acta Histochem., 115(1):3-7, 2013.

Yen, C. F.; Kim, S. H.; Liao, S. K.; Atabekoglu, C.; Uckac, S.; Arici, A.; Arlier, S.; Lee, C. L.; Wang, H. S. \& Kayisli, U. A. Increased expression of integrin-linked kinase during decidualization regulates the morphological transformation of endometrial stromal cells. Fertil. Steril., 107(3):803-12, 2017.

Yu, N.; Yan, W.; Yin, T.; Wang, Y.; Guo, Y.; Zhou, D.; Xu, M.; Ding, J. \& Yang, J. HCG-activated human peripheral blood mononuclear cells (PBMC) promote trophoblast cell invasion. PLoS One, 10(6): 0125589,2015

Zhang, D.; Ma, C.; Sun, X.; Xia, H. \& Zhang, W. S100P expression in response to sex steroids during the implantation window in human endometrium. Reprod. Biol. Endocrinol., 10:106, 2012.

Zhou, F.; Liang, Y.; Qian, Z. D.; Zhuang, Y. L.; Chen, Y. Z.; Lv, B. J.; Zhou, C. Y.; Chen, X. D. \& Huang, L. L. Low-dose mifepristone increases uterine expression of aquaporin 1/aquaporin 2 at the time of implantation. Contraception, 87(6):844-9, 2013.

\author{
Dirección para correspondencia \\ Ruth Prieto Gómez \\ Departamento de Pediatría y Cirugía Infantil \\ Facultad de Medicina \\ Universidad de La Frontera \\ Temuco \\ CHILE
}

E-mail: ruth.prieto@ufrontera.cl

Recibido : 22-05-2018

Aceptado: 27-11-2018 